\title{
A Cooperative UAV-Based Communications Backbone for Sensor Networks
}

\author{
R. S. Roberts
}

October 3, 2001

Lawrence

Livermore

National

Laboratory

U.S. Department of Energy

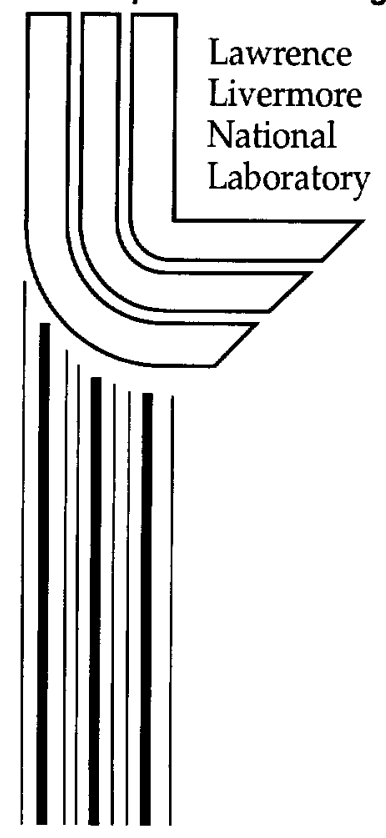




\section{DISCLAIMER}

This document was prepared as an account of work sponsored by an agency of the United States Government. Neither the United States Government nor the University of California nor any of their employees, makes any warranty, express or implied, or assumes any legal liability or responsibility for the accuracy, completeness, or usefulness of any information, apparatus, product, or process disclosed, or represents that its use would not infringe privately owned rights. Reference herein to any specific commercial product, process, or service by trade name, trademark, manufacturer, or otherwise, does not necessarily constitute or imply its endorsement, recommendation, or favoring by the United States Government or the University of California. The views and opinions of authors expressed herein do not necessarily state or reflect those of the United States Government or the University of California, and shall not be used for advertising or product endorsement purposes.

This work was performed under the auspices of the U. S. Department of Energy by the University of California, Lawrence Livermore National Laboratory under Contract No. W-7405-Eng-48.

This report has been reproduced directly from the best available copy.

Available electronically at http://www.doc.gov/bridge

Available for a processing fee to U.S. Department of Energy

And its contractors in paper from

U.S. Department of Energy

Office of Scientific and Technical Information

P.O. Box 62

Oak Ridge, TN 37831-0062

Telephone: (865) 576-8401

Facsimile: (865) 576-5728

E-mail: reports@adonis.osti.gov

Available for the sale to the public from

U.S. Department of Commerce

National Technical Information Service 5285 Port Royal Road Springfield, VA 22161

Telephone: (800) 553-6847

Facsimile: (703) 605-6900

E-mail: orders@ntis.fedworld.gov

Online ordering: http://www.ntis.gov/ordering.htm

OR

Lawrence Livermore National Laboratory

Technical Information Department's Digital Library

http://www.llnl.gov/tid/Library.html 


\title{
A Cooperative UAV-based Communications Backbone for Sensor Networks
}

\author{
3 October 2001
}

\begin{abstract}
This white paper describes an ongoing research project at the Lawrence Livermore National Laboratory in the area of sensor and communications networks. The contents of this white paper are preliminary, and are subject to change as the project evolves.
\end{abstract}

The objective of this project is to investigate the use of unmanned air vehicles (UAVs) as mobile, adaptive communications backbones for ground-based sensor networks. In this type of network, the UAVs provide communication connectivity to sensors that cannot communicate with each other because of terrain, distance, or other geographical constraints. In these situations, UAVs provide a vertical communication path for the sensors, thereby mitigating geographic obstacles often imposed on networks. With the proper use of UAVs, connectivity to a widely disbursed sensor network in rugged terrain is readily achieved.

Our investigation has focused on networks where multiple cooperating UAVs are used to form a network backbone. The advantage of using multiple UAVs to form the network backbone is parallelization of sensor connectivity. Many widely spaced or isolated sensors can be connected to the network at once using this approach. In these networks, the UAVs logically partition the sensor network into sub-networks (subnets), with one UAV assigned per subnet. Partitioning the network into subnets allows the UAVs to service sensors in parallel thereby decreasing the sensor-tonetwork connectivity. A UAV services sensors in its subnet by flying a route (path) through the subnet, uplinking data collected by the sensors, and forwarding the data to a ground station. An additional advantage of using multiple UAVs in the network is that they provide redundancy in the communications backbone, so that the failure of a single UAV does not necessarily imply the loss of the network.
The difficulty with constructing such a network is the design of algorithms to control and coordinate the operations of the UAVs. Our algorithm development efforts have focused on three classes of algorithms: 1) globally optimum algorithms that create subnets and find UAV routes through the subnets using global sensor position information; 2) locally optimum algorithms that modify subnets and paths through the subnets using locally derived connectivity and sensor state estimates; and 3) algorithms to search for lost sensors. The results of our investigation into the first class of algorithms is reported in [1]. Work on the other two classes of algorithms is ongoing.

As noted above, the purpose of UAVs in this network is to provide physical connectivity between sensors and a ground station. Once physical connectivity is established, performance of the network protocol stack becomes important. For a variety of reasons, we have elected to design the network using the Internet Protocol and IEEE 802.11 wireless standards. To study the performance of these protocols in this network, we are performing packet-level simulations of the network, using connectivity patterns generated by the movements of the UAVs.

To support our investigation, we have developed a software tool called STOMP, for Simulation, Tactical Operations and Mission Planning. STOMP maintains and displays the states of objects (e.g., sensors and UAVs) over a Digital Terrain Elevation Data (DTED) background (see Figure 1 below). An event queue associated with each object processes events by either time stamp or priority, thereby allowing external 
events (such as state updates from a real UAV or sensor) to be processed with simulated events (state updates from a simulated UAV or sensor). A message handler associated with each object determines the appropriate action for each type of event. Furthermore, the code is designed with an object hierarchy that allows for the easy addition of new features and algorithms.

As a final point, we are exploring the application of the UAV control, adaptation and communications techniques developed in this project to other sensing problems. In our current paradigm, UAVs fly to a waypoint (i.e., the location of a ground sensor), collect data, then fly to the next point. As the data is collected, it is relayed through the network to a ground station. By equipping the UAVs with appropriate sensors, and selecting a set of way-points as regions-ofinterest, the techniques developed here could be used by a group of UAVs to

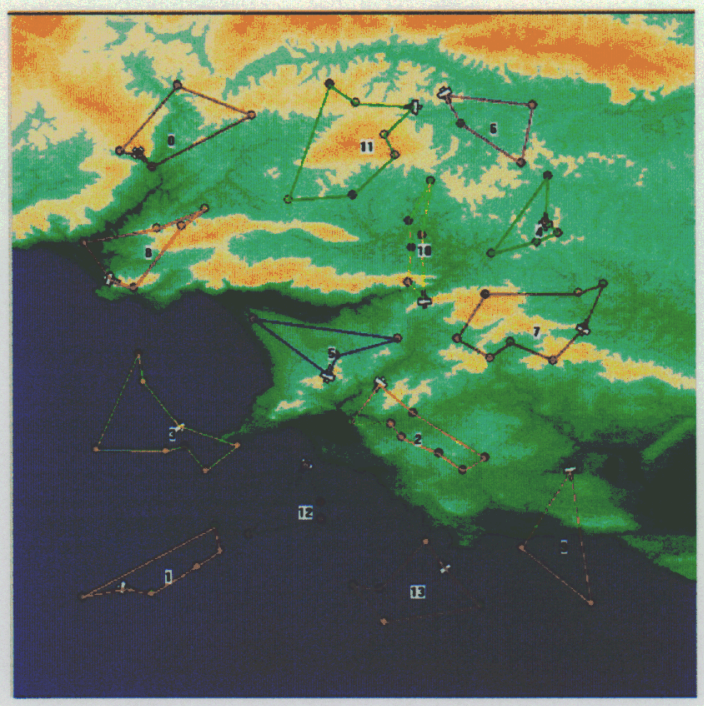

Figure 1: STOMP console illustrating a network of 14 UAVs servicing 79 sensors. The subnets are indicated by the polygons connecting groups of sensors. The subnets and path arrangements were produced by the algorithm reported in [1]. perform other missions such as coordinated aerial surveillance. This and other applications are under consideration.

This work was performed under the auspices of the U.S. Department of Energy by Lawrence Livermore National Laboratory under Contract W-7405-Eng-48.

For more information on this project, please contact Randy Roberts at the Lawrence Livermore National Laboratory, (925) 4239255 or at roberts38@llnl.gov.

\section{Reference:}

[1] C. Cunningham and R. Roberts, "An Adaptive Path Planning Algorithm for Cooperating Unmanned Air Vehicles", Proceedings of the 2001 IEEE International Conference on Robotics and Automation, Seoul Korea, May 2001. 